PROCEEDINGS OF THE AMERICAN MATHEMATICAL SOCIETY

Volume 124, Number 9, September 1996

\title{
ON THE REALIZABILITY OF LEWY STRUCTURES
}

\author{
ABDELHAMID MEZIANI \\ (Communicated by Eric Bedford)
}

\begin{abstract}
We prove that a nondegenerate CR structure with signature
$(p, n-p)$ at $0 \in \mathbb{R}^{2 n+1}$ and with $n$ first integrals $z_{1}, \cdots, z_{n}$ satisfying

$d z_{1} \wedge d \bar{z}_{1} \wedge \cdots \wedge d z_{n} \wedge d \bar{z}_{n} \neq 0$
\end{abstract}

is realizable if and only if an action of the group $O(2 p, 2 n-2 p)$ leaves invariant

a one-dimensional subbundle of the structure bundle.

The local realizability (embeddability) of $\mathrm{CR}$ structures has been the focus of several papers, among others we mention $[\mathrm{Ak}],[\mathrm{AH}],[\mathrm{BR}],[\mathrm{Ha}],[\mathrm{HJ}],[\mathrm{Ja} 1]$, [JT1, JT2, JT3, Ku, Ni]. It has been proved ([AH]) that abstract real analytic CR structures are realizable. It is also proved ([Ak], $[\mathrm{Ku}])$ that $C^{\infty}$ strictly pseudoconvex $\mathrm{CR}$ structures in $\mathbb{R}^{2 n+1}$ with $n \geq 3$ are embeddable. On the other hand, there exist nonrealizable $\mathrm{CR}$ structures: of strictly pseudoconvex nature in $\mathbb{R}^{3}$ ([Ni, JT1, JT2, JT3]); of Lewy type with signature $(1, n-1)$ in $\mathbb{R}^{2 n+1}$ ([JT1, JT2, JT3]); and of high codimension ([M1]).

In this note, we consider the realizability problem for a class of Lewy structures of hypersurface type at $0 \in \mathbb{R}^{2 n+1}$ with signature $(p, n-p)$. More precisely, we consider those structures with one first integral not listed. We prove that the "missing" first integral exists if and only if an action of the group $O(2 p, 2 n-2 p)$ leaves invariant a 1-dimensional subbundle of the Lewy bundle.

2

A Lewy structure at $0 \in \mathbb{R}^{2 n+1}$ with signature $(p, n-p)$ is the datum of a subbundle $\mathcal{C}$ of $\mathbb{C} T^{*} U$, where $U$ is a neighborhood of $0 \in \mathbb{R}^{2 n+1}$ and $\mathbb{C} T^{*} U$ is the complexified cotangent bundle $T^{*} U$, such that $\mathcal{C}$ is generated by $n+1 \operatorname{smooth} C^{\infty}$ one-forms $\omega_{1}, \cdots, \omega_{n+1}$ satisfying

$$
\begin{gathered}
\omega_{1} \wedge \cdots \wedge \omega_{n} \wedge \bar{\omega}_{1} \wedge \cdots \wedge \bar{\omega}_{n} \wedge \omega_{n+1} \neq 0, \\
\omega_{1} \wedge \cdots \wedge \omega_{n+1} \wedge d \omega_{k}=0 \quad \text { for } \quad k=1, \cdots, n+1,
\end{gathered}
$$

and such that the Levi form has $p$ positive and $n-p$ negative eigenvalues. It follows from the formal integrability and the nondegeneracy of the Levi form that in suitable coordinates $\left(x_{1}, y_{1}, \cdots, x_{n}, y_{n}, u\right)$ of $\mathbb{R}^{2 n+1}$ the second jets of $\omega_{1}, \cdots, \omega_{n+1}$

Received by the editors December 8, 1994 and, in revised form, March 10, 1995.

1991 Mathematics Subject Classification. Primary 32F25; Secondary 53C40.

(c)1996 American Mathematical Society 
are given by

$$
\begin{aligned}
j^{2} \omega_{k} & =d z_{k}, \quad \text { for } \quad k=1, \cdots, n, \\
j^{2} \omega_{n+1} & =d u+i\left(\sum_{l=1}^{p} z_{l} d \bar{z}_{l}-\sum_{l=p+1}^{n} z_{l} d \bar{z}_{l}\right),
\end{aligned}
$$

where $z_{k}=x_{k}+i y_{k}$. The Lewy structure is said to be realizable if the bundle $\mathcal{C}$ is generated by exact forms. (For a comprehensive treatment of CR structures we refer to $[\mathrm{Ja} 2]$ and $[\mathrm{Tr}]$.

In this note, we are concerned with those Lewy structures $\mathcal{L}$ with signature $(p, n-p)$ generated, in suitable coordinates, by the forms

$$
\begin{aligned}
\omega_{k} & =d z_{k} \quad \text { for } \quad k=1, \cdots, n, \\
\omega_{n+1} & =d u+\sum_{l=1}^{n} \lambda_{l} d \bar{z}_{l} .
\end{aligned}
$$

Hence $\omega_{n+1}$ satisfies

$$
d \omega_{n+1} \wedge d z_{1} \wedge \cdots \wedge d z_{n} \wedge \omega_{n+1}=0
$$

and its coefficients $\lambda_{l}$ satisfy

$$
\lambda_{l}=i \epsilon_{l} z_{l}+o\left(|(z, u)|^{2}\right),
$$

with $\epsilon_{l}=1$ if $l \leq p$ and $\epsilon_{l}=-1$ if $l \geq p+1$. Notice that in order for the Lewy structure $\mathcal{L}$ to be realizable, there must be $C^{\infty}$ functions $\mu_{1}, \cdots, \mu_{n}$ such that

$$
\omega_{n+1}+\mu_{1} d z_{1}+\cdots+\mu_{n} d z_{n}=g d f
$$

for some $C^{\infty}$ functions $f$ and $g$. The function $f$ is called ([Ha], [HJ]) the missing first integral of $\mathcal{L}$.

When the missing first integral $f$ exists, the form $d f$ defines a Mizohata structure with signature $|2 n-4 p|$ at $0 \in \mathbb{R}^{2 n+1}$ (see [Tr] and [M2] for details about Mizohata structures). We know from $[\operatorname{Tr}]$ that there exists a germ of a diffeomorphism $\Phi$ at $0 \in \mathbb{R}^{2 n+1}$, tangent to the identity $(D \Phi(0)=I)$, such that if $\Phi=\left(\phi_{1}, \cdots, \phi_{2 n+1}\right)$ and

$$
\begin{aligned}
t_{k} & =\phi_{k}(x, u) \quad \text { for } \quad k=1, \cdots, 2 n, \\
s & =\phi_{2 n+1}(x, u)
\end{aligned}
$$

then

$$
f \circ \Phi^{-1}(t, s)=s+i\left(\sum_{k=1}^{2 p} t_{k}^{2}-\sum_{k=2 p+1}^{2 n} t_{k}^{2}\right) .
$$

Hence the invariance group $O(2 p, 2 n-2 p)$ of the quadratic form

$$
q(t)=\sum_{k=1}^{2 p} t_{k}^{2}-\sum_{k=2 p+1}^{2 n} t_{k}^{2}
$$

acting on $\mathbb{R}^{2 n+1}$ by

$$
A(t, s)=(A t, s)
$$


leaves invariant the bundle generated by $d\left(f \circ \Phi^{-1}\right)$. Therefore the action of the group

$$
G=\Phi^{-1} O(2 p, 2 n-2 p) \Phi
$$

leaves invariant the one-dimensional subbundle of $\mathcal{L}$ generated by $d f$.

4

Conversely, we prove that the existence of a group $G$, conjugate to $O(2 p, 2 n-2 p)$ under a diffeomorphism $\Phi$ with $D \Phi(0)=I$, and the existence of a one-dimensional subbundle $\mathcal{T}$ of the Lewy bundle $\mathcal{L}$ such that

$$
G^{*} \mathcal{T}=\mathcal{T}
$$

is a sufficient condition for the realizability of the Lewy structure $\mathcal{L}$.

Suppose that $\mathcal{T}$ satisfying (8) is spanned by a form

$$
\omega=a \omega_{n+1}+\sum_{k=1}^{n} b_{k} d z_{k} .
$$

We claim that $a(0) \neq 0$ and $b_{k}(0)=0$ for $k=1, \cdots, n$. Indeed, let $A \in$ $O(2 p, 2 n-2 p)$ be defined by

$$
A\left(t_{1}, \cdots, t_{2 n}, s\right)=\left(t_{1},-t_{2}, \cdots, t_{2 n-1},-t_{2 n}, s\right) .
$$

Since $D \Phi(0)=I$, then $\Psi=\Phi^{-1} \circ A \circ \Phi$ satisfies

$$
\Psi\left(x_{1}, y_{1}, \cdots, x_{n}, y_{n}, u\right)=\left(x_{1},-y_{1}, \cdots, x_{n},-y_{n}, u\right)+o(|(z, u)|) .
$$

Thus,

$$
\Psi^{*} \omega(0)=a(0) d u+\sum_{k=1}^{n} b_{k}(0) d \bar{z}_{k}
$$

This shows that for $\Psi^{*} \omega$ to be a section of $\mathcal{T}$, it is necessary to have $a(0) \neq 0$ and $b_{k}(0)=0$ as claimed.

After dividing $\omega$ by $a$, we see that $\mathcal{T}$ is spanned by a form

$$
\begin{aligned}
\Omega & =\omega_{n+1}+\sum_{k=1}^{n} \mu_{k} d z_{k} \\
& =d u+\sum_{k=1}^{n} \lambda_{k} d \bar{z}_{k}+\sum_{k=1}^{n} \mu_{k} d z_{k} .
\end{aligned}
$$

Now, observe that since

$$
\Psi^{*} \Omega \wedge \Omega=0,
$$

( $\Psi$ is defined in (10)) and since the $\lambda_{k}$ 's satisfy (4), it follows that necessarily

$$
\mu_{k}=i \epsilon_{k} \bar{z}_{k}+o\left(|(z, u)|^{2}\right)
$$

This means that the first jet of $\Omega$ is

$$
j^{1} \Omega=d u+i \sum_{k=1}^{n} \epsilon_{k} d\left(z_{k} \bar{z}_{k}\right) .
$$

With this, our aim would be acheived if we could show that

$$
\Omega \wedge d \Omega=0 .
$$


For then $\Omega$ would generate a Mizohata structure with signature $|2 n-4 p|$ at $0 \in$ $\mathbb{R}^{2 n+1}$ and we know from [M2] that such a structure is locally integrable, i.e.,

$$
\Omega=g d f .
$$

The function $f$ would then be the missing first integral of the Lewy structure $\mathcal{L}$.

To prove the formal integrability (16), we use again the change of coordinates $\Phi$ and write

$$
\left(\Phi^{-1}\right)^{*} \Omega=\alpha(t, s)\left[d s+\sum_{k=1}^{2 n} a_{k}(t, s) d t_{k}\right]=\alpha(t, s) \Theta .
$$

Since the bundle generated by the form $\Theta$ is invariant under the action of the group $O(2 p, 2 n-2 p)$, then it is not difficult to see that

$$
A^{*} \Theta=\Theta \quad \forall A \in O(2 p, 2 n-2 p) .
$$

To prove that $\Theta$ is formally integrable, it is enough to prove that it descends to the orbit space $\mathbb{R}^{2 n+1} / O(2 p, 2 n-2 p)$ as a form $\bar{\Theta}$. The latter form would, necessarily, be formally integrable because of the dimension two of the orbit space. More precisely, we prove the following lemma.

Lemma. Let $q(t)$ be the quadratic form defined in (6) and $\Theta$ the 1-form defined in (18) and satisfying (19). Then, in each connected component of $\mathbb{R}^{2 n+1} \backslash\{q(t)=0\}$ we have

$$
\Theta=d s+g(q(t), s) d(q(t))
$$

for some function $g$ of two variables.

Proof. Consider the spherical coordinates

$$
\begin{aligned}
\Pi_{1}: \mathbb{R} \times S^{2 p-1} \longrightarrow \mathbb{R}^{2 p}, & \Pi_{1}\left(r_{1}, \theta^{1}\right)=r_{1} \theta^{1}, \\
\Pi_{2}: \mathbb{R} \times S^{2 n-2 p-1} \longrightarrow \mathbb{R}^{2 n-2 p}, & \Pi_{2}\left(r_{2}, \theta^{2}\right)=r_{2} \theta^{2} .
\end{aligned}
$$

We have then

$$
\Theta=d s+U_{1} d r_{1}+U_{2} d r_{2}+\Lambda
$$

in $s, r_{1}, r_{2}, \theta^{1}, \theta^{2}$ coordinates, where $U_{1}, U_{2}$ are functions and $\Lambda$ is a 1 -form on $S^{2 p-1} \times S^{2 n-2 p-1}$ with $s, r_{1}, r_{2}$ as parameters.

Let $R^{1}$ and $R^{2}$ be arbitrary rotations of $S^{2 p-1}$ and $S^{2 n-2 p-1}$, respectively. It follows from (19) that

$$
U_{k}\left(s, r_{1}, r_{2}, R^{1} \theta^{1}, R^{2} \theta^{2}\right)=U_{k}\left(s, r_{1}, r_{2}, \theta^{1}, \theta^{2}\right), \quad k=1,2 .
$$

Since the group of rotations is transitive on the sphere, then $U_{1}$ and $U_{2}$ are independent of $\theta^{1}, \theta^{2}$. Now we are going to prove that in a connected component of $\mathbb{R}^{2 n+1} \backslash\{q=0\}$ we have

$$
U_{1}\left(s, r_{1}, r_{2}\right) d r_{1}+U_{2}\left(s, r_{1}, r_{2}\right) d r_{2}=g\left(r_{1}^{2}-r_{2}^{2}, s\right) d\left(r_{1}^{2}-r_{2}^{2}\right)
$$

for some function $g$ of two variables. For this, we consider the hyperbolic radius $\rho^{2}=r_{1}^{2}-r_{2}^{2}$ and the change of variables

$$
r_{1}=\rho \cosh t, \quad r_{2}=\rho \sinh t .
$$

In the coordinates $(\rho, t)$ the form $\Gamma=U_{1} d r_{1}+U_{2} d r_{2}$ has the expression

$$
\begin{aligned}
\Gamma & =\left(U_{1} \cosh t+U_{2} \sinh t\right) d \rho+\rho\left(U_{1} \sinh t+U_{2} \cosh t\right) d t \\
& =V d \rho+\rho W d t .
\end{aligned}
$$


From (19), it follows that $\Gamma$ is invariant under the translations $t \mapsto t+\tau$ and under the reflection $t \mapsto-t$. The invariance under translations implies that $V$ and $W$ are independent of $t$, and then the invariance under the reflection gives $W=0$. This proves (23).

To complete the proof of the lemma, we go back to (21) and prove that $\Lambda=0$. Again from (19) it follows that $A^{*} \Lambda=\Lambda$ for every $A \in O(2 p, 2 n-2 p)$. Let $\Lambda=\Lambda_{1}+\Lambda_{2}$, where $\Lambda_{1}$ is a 1 -form on $S^{2 p-1}$ with parameters $s, r_{1}, r_{2}, \theta^{2}$ and $\Lambda_{2}$ is a form on $S^{2 n-2 p-1}$ with parameters $s, r_{1}, r_{2}, \theta^{1}$. We deduce that

$$
A_{1}^{*} \Lambda_{1}=\Lambda_{1} \quad \text { and } \quad A_{2}^{*} \Lambda_{2}=\Lambda_{2}
$$

for every $A_{1} \in O(2 p)$ and every $A_{2} \in O(2 n-2 p)$.

Now suppose that $\alpha=a(x, y) d x+b(x, y) d y$ is a 1 -form in a two-dimensional plane satisfying $B^{*} \alpha=\alpha$ for every $B \in O(2)$. We write $\alpha$ in polar coordinates $(r, \phi)$ as

$$
\begin{aligned}
\alpha & =(a \cos \phi+b \sin \phi) d r+r(-a \sin \phi+b \cos \phi) d \phi \\
& =p(r, \phi) d r+r q(r, \phi) d \phi .
\end{aligned}
$$

Since $\alpha$ is invariant under the translations $\phi \mapsto \phi+\tau$, it follows at once that $p$ and $q$ are independent of $\phi$. The invariance of $\alpha$ under the reflection $\phi \mapsto-\phi$ shows that $q=0$. That is,

$$
\alpha=p(r) d r .
$$

With this at hand, we prove that $\Lambda_{1}=0$ as follows. Take any point $p_{0} \in S^{2 p-1}$ and consider the stereographic projection with center $p_{0}$. Consider the action of the group $O(2)$ on the chart $\mathbb{R}^{2 p-1}=\mathbb{R}^{2} \times \mathbb{R}^{2 p-3}$, with $p_{0}$ as the origin, as $\left(x, x^{\prime}\right) \mapsto$ $\left(B x, x^{\prime}\right)$. The form $\Lambda_{1}$ is invariant under this action and so it follows from (28) that $\Lambda_{1}$ is independent of the polar angle $\phi$ in $\mathbb{R}^{2}$. Since $p_{0}$ is arbitrary in $S^{2 p-1}$, the nullity of $\Lambda_{1}$ follows. Similarly we prove that $\Lambda_{2}=0$. The lemma is proved.

In conclusion, we have proved the following

Theorem. A Lewy structure $\mathcal{L}$ with signature $(p, n-p)$ and with only one "missing" first integral is realizable if and only if there exist a germ of a diffeomorphism $\Phi$ at $0 \in \mathbb{R}^{2 n+1}$, tangent to the identity, and a 1-dimensional subbundle $\mathcal{T}$ of the Lewy bundle such that

$$
\Psi^{*} \mathcal{T}=\mathcal{T} \quad \forall \Psi \in \Phi^{-1} O(2 p, 2 n-2 p) \Phi .
$$

\section{REFERENCES}

[Ak] T. Akahori, A new approach to the local embedding of CR structures for $n \geq 4$ (the local solvability of the operator $\bar{\partial}_{b}$ in the abstract sense), vol. 366, Memoirs Amer. Math. Soc., 1987. MR 88i:32027

$[\mathrm{AH}]$ A. Andreotti and C.D. Hill, Complex characteristic coordinates and tangential Cauchy Riemann equations, Ann. Scuola Norm. Pisa 26 (1972), 299-324. MR 57:717

[BR] M.S. Baouendi and L.P. Rothschild, Embeddability of Abstract CR structures and integrability of related systems, Ann. Inst. Fourier Grenoble 37 (1987), 131-141. MR 89c:32053

[Ha] N. Hanges, The missing first integral, J. Diff. Equations 72 (1988), 178-188. MR 89j:32016

[HJ] N. Hanges and H. Jacobowitz, A remark on almost complex structures with boundary, Amer. J. Math 111 (1989), 53-64. MR 90m:32038

[Ja1] H. Jacobowitz, The canonical bundle and realizable of CR hypersurfaces, Pacific J. Math. 127 (1987), 91-101. MR 88e:32027

[Ja2] - An introduction to CR structures, Math Surveys and Monographs, vol. 32, Amer. Math. Soc, 1990. MR 93h:32023 
[JT1] H. Jacobowitz and F. Treves, Non-realizable CR structures, Invent. Math. 66 (1982), 231249. MR 83f: 53022

[JT2] — Aberrant CR structures, Hokkaido Math. J. 12 (1983), 267-292. MR 85c:32033

[JT3] Nowhere solvable homogeneous partial differential equations, Bull. Amer. Math. Soc. 8 (1983), 467-469. MR 85b:32031

[Ku] M. Kuranishi, Strongly pseudoconvex CR structures over small balls, I-II-III, Ann. of Math. 115 - 116 (1982), 451-500; 1-64; 249-330. MR 84h:32023c

[M1] A. Meziani, Perturbation of a class of CR structures of codimension larger than one, J. Funct. Analysis 116 (1993), 225-244. MR 94i:32024

[M2] Classification of germs of Mizohata structures, Comm. PDE 20 (3-4) (1995), 499539. CMP 95:08

[Ni] L. Nirenberg, Lectures on linear partial differential equations, Reg. Conf. Series in Math., vol. 17, Amer. Math. Soc., 1973. MR 56:9048

[Tr] F. Treves, Hypo-analytic structures: local theory, vol. 40, Princeton University Press, 1992. MR 94e:35014

Department of Mathematics, Florida International University, Miami, Florida 33199

E-mail address: meziani@servax.fiu.edu 